\title{
STRATEGIC ACTIVITY PLANNING FOR EMOTIONALLY DISTURBED CHILDREN AND ADOLESCENTS
}

\author{
Andrew J. Schneider-Muñoz, \\ University of Pittsburg
}

With kind permission from the: Journal of Child and Youth Care Work, Strategic Activity Planning for Emotionally Disturbed Children and Adolescents, Vol. 4, Summer 1988, 71-79, Schneider-Muñoz, A. J., (C) National Organization of Child Care Worker Associations, Inc.

\begin{abstract}
Strategically planned activity components for residential treatment program directly reduced the amount of negative acting out which must be managed in the cottage environment. Sequentially guided creative tasks can provide a supportive central core for treatment goal implementation. Organized activities serve to measure progress towards individual and peer relationship objectives. Specific step-by-step strategies and projects are recommended for use within the therapeutic milieu for emotionally disturbed children and adolescents.
\end{abstract}

"There is always one moment in childhood when the door opens and lets the future in."

\section{"The Power and the Glory" \\ Graham Greene}

\begin{abstract}
"One of the most painful things in the Western United States and Territories is the extinction of childhood. I have never seen any children, only debased imitations of men and women, cankered by greed and selfishness, and asserting and gaining complete independence of their parents at ten years old."
\end{abstract}

\author{
"A Lady's Life in the Rocky Mountains" \\ Isabella L. Bird (1873)
}

This article is dedicated to Marvin, who started out eating the crayons and is now an artist.

As child care professionals, how often have we made the well-intentioned trip to the local public library or teachers' supply store only to find a plethora of activity resources for mainstream children. These projects present, at best, tasks which are too complex for the emotionally disturbed child with limited attention span and low impulse control. At their worst, these materials offer challenges which can create frustrating situations for children lacking internalized controls and cognitive boundaries. 
Somewhere in between the range of clinical art therapy and traditional recreation, line workers are often asked to "keep the kids busy." How important is a structured activity program established within the residential treatment milieu? Do these activities support, enhance, or constitute a valid modality for treatment goal implementation? This article will attempt to address these issues, as well as offer practical strategies to be utilized within the therapeutic milieu.

The flow of residential treatment pivots around community meetings, crisis intervention, situational teaching within the context of everyday life skills, and individual clinical sessions. There is a dynamic tension in setting limits to manage behavioral objectives. We are losing half the battle if we let our treatment teams slip into the headset of reacting to these demands by attempting to "let the kids sandwich in some play time to get their energy out," almost in conflict with what's next on the schedule.

For the most part, the emotionally disturbed children in our residential facilities are "sweepstakes kids." They could easily win the grand prize for evading the intervention of a large number of talented community professionals including teachers, principals, therapists, parole officers, and next door neighbors. The placement of a child in a costly highly structured setting represents that the community has reached its tolerance level in the ability to meet the child's needs.

These challenging children must be kept safe as we support them in confronting their growth through treatment. Line workers offer their expertise in generating and maintaining the therapeutic structure which keeps us"one step ahead of the kids."

Let's be practical. The high turnover rate in our field tends to suggest that we are overworked and underpaid. Without more staff and more funding, how do we add activities on top of what we already do?

A carefully organized program can directly reduce the amount of acting out generated in any given shift. These activities can be designed to accomplish treatment objectives. Sequentially guided creative tasks which are successfully completed serve as one key to the investment process. They are a productive vehicle to conduct the formation of the elusive concepts of building self-esteem, developing interactional skills, and establishing behavioral controls.

A little creative energy in thinking through an effective activity component can provide a supportive central core for the entire treatment program conducted within the milieu. Children respond to the idea that we are moving forward together to the next activity which has already been planned. Activities become a ritualized and valued part of the cottage culture. Such an approach moves away from simply "maintaining" children which in the end produces anxiety for everyone involved. Play activities can serve as a mark with which to measure progress toward individual and peer relationship objectives.

Working together with child care counselors, I have constructed activity components within the residential treatment milieu for severely emotionally disturbed, 
autistic, and delinquent children and adolescents. I would encourage you to draw from the following steps:

1. Adults stopping for even a short time to mull over ideas for the next appropriate activity can lose the positive momentum for the children during a shift. Even the time it takes to search out a few pencils can leave enough space for acting out. Plan ahead and gather your resources beforehand. Materials should be readily available.

2. Plan a series of activities with different formats and varying degrees of complexity. This allows the team to check the mood of the group and respond rapidly with the most appropriate activity for the condition of the milieu.

3. Evaluate the strengths and talents of your existing team. Identify activities which the children can accomplish that staff enjoy. Providing staff with a supportive environment with which to be creative and to integrate their individual skills into the program significantly increases commitment and reduces burnout.

4. Start slowly and simply with activities. Ask the children for their involvement in planning by presenting several options, rather than asking for a yes-no response. Seek out the natural leaders of the children's own status hierarchy and win over their positive peer support.

5. Size up your physical environment and select a space which can be clearly designated as an activity center within the cottage culture. The library, the corner of the living room, or changing furniture in just about any room will do. Walls to display completed projects are essential. A space you can "mess up" even for a short time is preferable to an environment which needs a lot of don'ts."

6. It is absolutely essential to extend the previously agreed upon limits and behavioral expectations of the milieu into the planned activity. Children are secure in knowing ground rules, the place for a time out, and the consequences for behavior. Hold a group and reaffirm these standards by getting the children to repeat their understanding of the basic framework.

7. Reinforcement and recognition supports development of an activities component targeted for strongly individualized child investment. Our culture tends to socialize merit. You might start with competitive language such as "best project wins a prize" to get an initial response from the children, but actually compensate for inclusive involvement by giving a reward to each member of the group; i.e.,"best coloring, best idea, best concentration," etc. It is usually most effective to give reinforcements which have al- 
ready been established within the milieu. You might also consider offering colored pencils, markers, tracing paper, etc. as recognition for effort which leads to the next project. Low impulse control children may need immediate reinforcement for step by-step task completion, as well as recognition for the finished product.

Imagination is far more important than large funding sources. Survey the cottage environment and put to work what you already have. Make it a cottage project to fix old furniture to provide work spaces. A stack of magazines can become collages. Save boxes and containers from the kitchen. Depending upon your agency's policy, you might want to check the local community for donated resources. I have found social services groups and store managers particularly helpful as long as you can articulate a clear need which they can support within their donation process. It has also been my experience that children make a statement about the value of activity projects by eventually volunteering their own funds. Of course, the primary responsibility belongs to the agency, but child-initiated support within appropriate limits completes the cooperative process.

Unlike educational settings which require a set curriculum, I have often valued the flexibility of the residential treatment milieu. If you plan a project which isn't working, stop and shift your strategy to the next task at which the children can feel a sense of success. After rethinking your approach or there is a shift in the contextual mood of the cottage environment, you can always make a second pass at completing the first idea at a different time.

The key to strategic activity planning is to select a range of projects with which the children can feel a sense of immediate accomplishment. Grand designs often fulfill adult or overall program expectations, but can leave children with a disrupted feeling. The following strategies take the vantage point of high reward and low frustration:

Before introducing the activity project, think through the specific abilities and stressors for each child. Select an engagement pattern which the adult works with children in dyads or triads depending on tactile and peer relationship skills. The adult can call the children to the activity area one group after another.

As the activity component expands within the cottage culture, the number of children working at one time can be increased as the interactional ground rules become ritualized and as peers can be cultivated to take a leadership role.

We all might complete the same project, but the project may be laid out in twelve different ways. Break the project down into a series of steps based upon skill level highly tailored to the individual child. For example, one child can handle utilizing scissors effectively. Another child may need all of the parts pre-cut and arranged in a pile. For the second child described, the simple act of attempting to cut could cause him/her to shut down in frustration. The scissors set up a situation predisposed to negative acting out. 
In adapting activity projects from resources designed for mainstream children, it is crucial for adults to control the tools which are difficult to utilize in a residential treatment setting. When working with a group, I often place these items out of reach on a high shelf, unless they are in my hands. Just as we keep a close eye on kitchen knives, it is important to keep a careful inventory of your equipment. Keeping track averts disaster in a preventive mode.

Don't be afraid to interrupt successful activity. We work so hard and long to encourage children to have productive play that when they do, we have a tendency to let the activity coast and continue beyond the children's ability to handle the situation.

It may be a major victory for one child to focus and concentrate enough to be able to glue two parts together. If a child succeeds with a difficult task, sense out his/ her ability to forge ahead. Rather than allowing the child to blow out his/her progress, end the completed task, provide praise with reinforcement, and make plans to do the next step at a future time. In this way a child begins to look forward to future success.

Organized play activities are often shuffled into the low priority category of budget and time permitting. Implement a strategic treatment purpose for these activities. The projects will take on an indispensable role within the operation of the cottage. Art, in particular, can draw the children into creating a symbolic representation of the concepts which we are encouraging them to internalize. These projects become a safe vehicle with which to release and communicate feelings. Strategically planned activities expand our ability to support the children in their practice of everyday life skills critical to a return to the community.

Play legos, draw a picture, do your chores, glue a model. How is this any different from what we did before? The next section of this article will address specific idea suggestions for project development.

1. Collections. If you can find even a single item which interests a hardto-reach child, you can begin an investing relationship. Together with children, I have collected pine cones, auto parts, fast food wrappers, war ribbons, etc. A runner has a harder time going out the door if she/ he first needs to pack her/his collection. We worked with a borderline child whose damage to cottage property pushed him towards needing to leave the program. We were able to invest him in collecting pictures of sports figures cut from old magazines. He would spend hours taping the pictures up in his room. We had a large supply of old sports magazines. He shifted his need to destruct from cottage property to these pictures. Changes in collections such as "disappearing" or "giving away" almost always signaled the advent of important treatment issues.

2. Cooking. Cooking is all in the attitude. It can be a burdening chore on top of child care or you can turn your kitchen into a learning laboratory which 
can occupy children for hours. Children gain a stake in providing for their own nurturance and nutrition. What a wonderful place to teach reading, following directions, measurement math, and group sharing. Create a family-like environment in the kitchen where it is safe to process feelings about past family gatherings. Hold a weekly special meal which encourages child choice and appropriate judgment. Also, menus offer a great opportunity to explore ethnic heritage.

3. Posters. Not only do brightly colored posters add a sense of child ownership to the environment, but these messages provide a symbolic projection for a focus on cottage treatment issues. A roll of white butcher paper can be one of the most important teaching tools you can invest in. Constructing posters provides a concrete task for group expression of ideas, thoughts, feelings. Graphic presentations reinforce the concepts we are encouraging the children to internalize.

4. Display. As the cottage culture strengthens, it becomes important for the child's externalized sense of positive self-accomplishment to appropriately display completed projects. Set aside a special set of shelves, a bookcase, or even a section of the cottage to exhibit these items. Secure their loan from the children to the group and then rotate these materials. At first we assumed such an approach would just provide a new target for children who act out. Instead, with positive peer pressure, the display areas rapidly gained a highly protected status within the children's culture. Serving as the curator for the display then became a project in itself. (Adequate storage for projects in process can be a great asset).

5. Draw. It's easy to fall into the pattern of attending to other tasks while children occupy their time filling pages of endless drawings. At the other extreme, we tend to over pathologize the meaning of drawings without the benefit of an art therapist's expertise. Children can often draw what they can't directly verbalize. It is important to get children to talk about the meaning of what they are drawing. After hours of formal treatment, I am often startled by how much more can be learned about a child's world by participating in a drawing session. Try a series of drawings encouraging children to describe the impressions of their own world: family, neighborhood, favorite play activity, etc. Drawings can also be utilized to evaluate the child's perceptions of the program by asking the group to draw their picture of outings, household tasks, consequences, etc. in a recorded social history format.

6. Garden. Gardening is relatively inexpensive compared to taking the entire group to a movie. The growth process requires daily cultivation, thus teaching the effort it takes to nurture with its visible rewards. Window 
boxes and buckets work in an urban setting. A large plot in a rural area can even augment the food budget. Here again, child success is the key factor. Pick hardy plants which grow easily in your climate and soil conditions. Too many tomatoes and squashes would be better than rows of seeds which don't come up. A good multiple season, with skill level expanding by the years. One child who had lived in fourteen different placements would bolt out the door every morning and say that he would not leave until his plants bloomed. Of course, by harvest he would continue in the program because he had given us the time and investment to make treatment progress. A trip to the garden center for each child who earns a new plant by meeting behavioral objectives can make a wonderful addition to the reinforcement list in your cottage.

7. Toys. As child care workers, we are always fighting against the growing clutter of the toys and personal belongings which are a given with children. I strongly recommend keeping these items to a manageable level by rotating these belongings into and out of storage space outside the center of the milieu. (Sealed trash bags or cardboard boxes in the basement are fine). Rethink the strategic use of toys. Encourage an afternoon of group play in which the children build a project together. (Lego castle, model car roadway, make believe town). Take a closet of broken board games and make it a cottage project to scour yard sales to refurbish these sets. Hold a board game competition which also gives rewards for group interactional skills.

8. Outings. Child-oriented programs are almost always on the go. These outings present a significant behavioral management challenge for line workers. I have found that the single most valuable strategy is to always know where you are going. Children key their security in a change of settings to the anxiety level of the adults. The focus should always be on supervising the children rather than figuring out the next move. When you are alone about the community in your off hours, scout out places to take the children. Five minutes sitting in a new park observing who goes there or introducing yourself to a local store manager can be worth its weight in gold if you must later manage acting out from children in public. Always review behavioral expectations before getting started by having the children repeat the rules. Each institution has its own unique set, but mine are: stick together, always ask, act out and you go back.

If our children are to leave institutional placement and eventually return to their community, we must remain committed to taking difficult children into public places. These situational exposures in realistic settings provide the opportunity to pattern appropriate behavior. Children enjoy the feel of spontaneity. You can make these outings without exposing the card 
that you are prepared by having been there first.

On a tour of a downtown high rise building, which the children enjoyed, one child threw himself on the floor at the 35th story refusing to use the elevator or walk the stairs. Thank goodness the business crew knew we were coming and did not overreact to severe acting out but, more important, what a crucial situation to teach trust in the ability of adults to take care of a scared and anxious child.

9. Theme. Theme provides a unifying force for the activity component which encourages staff to contribute by plugging in their ideas. Children are provided with a focused unit working on a targeted treatment issue in week or month long segments such as Friendship (peer relationships), Cultural Heritage (self-awareness), Family (relationship systems), Community (basic reintegration skills), Spring Cleaning (personal hygiene, cottage organization, etc.) Review individual treatment plans for frequently targeted therapeutic goals, then move forward toward a theme, developing activities which reflect these objectives. Depending upon client turnover rate, effective themes and activity implementation plans can be repeated in rotation.

It is just as important to consider the positive of what can be accomplished, balanced by preventive contemplation of what might go wrong. Strategically planned activities offer a strong asset for the concrete implementation and measure of treatment plan progress. Sequentially guided creative tasks provide step-by-step definition for individual and relationship objectives in a child-centered format.

Carefully planned activities tap the talent of the staff and at the same time provide a focal point for the children to internalize behavioral skills. Activities draw children and adults together in a shared relationship-building process which supports communication. Ritualized activity components within the cottage culture create a productive environment which moves away from the maintenance of children and reduces negative acting out.

A note of appreciation to the Edgewood Children's Center Residential Program which continues to support creative adventures in our cottage.

A special thanks to Bill Evans, Director of The Western Academy, Rifle, Colorado. 


\section{Sample Activities Schedule Six-Hour Implementation Plan}

SUNDAY

$45 \mathrm{~min}$.

MONDAY

$45 \mathrm{~min}$.

TUESDAY

75 min.

WEDNESDAY

45 min.

THURSDAY

$60 \mathrm{~min}$.

FRIDAY

60 min.

SATURDAY

$120 \mathrm{~min}$.
Each child returning from a home visit draws his favorite place to play during the weekend away. Activity utilized to conduct transition and group reintegration. Allows communication of feelings to the team and assessment for intervention need.

During weekly community meeting, encourage children to plan upcoming Special Meal. Stress appropriate judgment, teamwork, and taking responsibility.

Craft project. Complete making mobiles from shells, driftwood, and other items from last week's outing to the beach.

Design a wall banner which illustrates recently established cottage rules for riding bikes and skateboards. Reinforces and nonverbally externalizes behavioral expectation.

Copy and color flora and fauna from books checked out of the public library that describes the recreational area the children will visit next week. Previews outing as a project to look forward to and creates learning focus.

Outing to the market to purchase foods for Special Meal. Situational teaching about nutrition and practical math.

Children prepare and serve Special Meal. Create a familylike environment to teach relationship skills. Table discussion processes past negative and positive experiences with family gatherings. 